\title{
Lemierre Syndrome Demonstrating an Atypical Case of Streptococcus Constellatus Causing a Deep Vein Thrombosis in the Left Internal Jugular Vein
}

\author{
Christian H. Summa \\ Nova Southeastern University, cs2641@mynsu.nova.edu \\ Marcos A. Clavijo Fernandez \\ Nova Southeastern University, mc3449@mynsu.nova.edu \\ Jordan Simpson \\ Nova Southeastern University, js4566@mynsu.nova.edu \\ Andrew Beckler \\ Center for Advanced Facial Plastic, Reconstructive and Cosmetic Surgery, Otolaryngology Consultants, \\ adbeckler@gmail.com \\ Michelle Demory Beckler \\ Nova Southeastern University, mbeckler@nova.edu
}

Follow this and additional works at: https://nsuworks.nova.edu/ijahsp

Part of the Medicine and Health Sciences Commons

\section{Recommended Citation}

Summa CH, Clavijo Fernandez MA, Simpson J, Beckler A, Demory Beckler M. Lemierre Syndrome Demonstrating an Atypical Case of Streptococcus Constellatus Causing a Deep Vein Thrombosis in the Left Internal Jugular Vein. The Internet Journal of Allied Health Sciences and Practice. 2022 Jan 03;20(1), Article 9.

This Case Study is brought to you for free and open access by the College of Health Care Sciences at NSUWorks. It has been accepted for inclusion in Internet Journal of Allied Health Sciences and Practice by an authorized editor of NSUWorks. For more information, please contact nsuworks@nova.edu. 


\title{
Lemierre Syndrome Demonstrating an Atypical Case of Streptococcus Constellatus Causing a Deep Vein Thrombosis in the Left Internal Jugular Vein
}

\begin{abstract}
Lemierre Syndrome (LS) is a rare, potentially life-threatening infection that typically develops from invasion of bacteria through pharyngeal mucosal tissue, followed by septic thrombophlebitis, most often involving the internal jugular vein. ${ }^{1}$ The primary complication of concern is the formation of septic emboli to the lungs or other organs. Fusobacterium necrophorum is the most common causative pathogen of LS. ${ }^{1}$ Typically, patients present with high fevers, sore throat, neck pain, and pulmonary symptoms. Though LS diagnosis is often overlooked because the initial manifestations may be subtle and non-specific, prompt diagnosis and treatment with antibiotics are imperative to prevent disease progression and promote quick recovery.
\end{abstract}

\section{Author Bio(s)}

1. Christian Summa, M.B.S, is a third year medical student at Dr. Kiran C. Patel College of Osteopathic Medicine.

2. Marcos Clavijo Fernandez is a third year medical student at Dr. Kiran C. Patel College of Osteopathic Medicine.

3. Jordan Simpson is a second year medical student at Dr. Kiran C. Patel College of Osteopathic Medicine.

4. Andrew Beckler, M.D. works for Center for Advanced Facial Plastic, Reconstructive and Cosmetic Surgery, Otolaryngology Consultants.

5. Michelle Demory Beckler Ph.D.is the Director of the Masters of Biomedical Sciences. She is also an Assistant Professor, Division of Microbiology and Immunology for Dr. Kiran C. Patel College of Allopathic Medicine.

\section{Acknowledgements}

The authors wish to extend their thanks to Beth Gilbert for her contribution to this study. 


\title{
IIJAHSP \\ The Internet Joumnal of Allied Health Sciences and Practice \\ Dedicated to allied health professional practice and education \\ Vol. 20 No. 1 ISSN 1540-580X
}

\section{Lemierre Syndrome Demonstrating an Atypical Case of Streptococcus Constellatus Causing a Deep Vein Thrombosis in the Left Internal Jugular Vein}

\author{
Christian H. Summa ${ }^{1}$ \\ Marcos A. Clavijo Fernandez ${ }^{1}$ \\ Jordan Simpson ${ }^{1}$ \\ Andrew Beckler ${ }^{2}$ \\ Michelle Demory Beckler ${ }^{1}$
}

1. Nova Southeastern University

2. Center for Advanced Facial Plastic, Reconstructive,

and Cosmetic Surgery, Otolaryngology Consultants

United States

\begin{abstract}
Lemierre Syndrome (LS) is a rare, potentially life-threatening infection that typically develops from invasion of bacteria through pharyngeal mucosal tissue, followed by septic thrombophlebitis, most often involving the internal jugular vein. ${ }^{1}$ The primary complication of concern is the formation of septic emboli to the lungs or other organs. Fusobacterium necrophorum is the most common causative pathogen of LS. ${ }^{1}$ Typically, patients present with high fevers, sore throat, neck pain, and pulmonary symptoms. Though LS diagnosis is often overlooked because the initial manifestations may be subtle and non-specific, prompt diagnosis and treatment with antibiotics are imperative to prevent disease progression and promote quick recovery.
\end{abstract}

Keywords: Lemierre's Syndrome, Streptococcus constellatus, Streptococcus anginosus, oropharynx, thrombosis, internal jugular vein 


\section{CASE PRESENTATION}

The patient was a 40-year-old female current smoker with a past medical history of anxiety. She presented with a muffled voice, odynophagia, and bilateral neck swelling. She also presented with dysphonia and mild tenderness in the affected area. Patient attributed these symptoms to swallowing a hot meatball several days prior. Based on history and physical, the patient did not appear to have any respiratory distress, but there was erythema and induration present in the neck. A Computer Tomography (CT) of the neck was performed and a large fluid and gas collection emanated from the left hypopharynx. There was focal narrowing of the left jugular vein as it approached the process at the level hyoid bone. An ultrasound study of the neck was performed and confirmed a DVT within the left internal jugular vein. Moreover, a CT of the chest showed an inferior margin of the anterior neck collection extending just below the sternoclavicular junction level, yet the mediastinum was unremarkable. Based on the radiology reports, history and physical exam compiled, the patient underwent a direct laryngoscopy to repair the left pharyngeal injury with incision and drainage of the neck and upper chest abscess.

\section{BACKGROUND}

What was once called, "The Forgotten Disease," LS is a rare condition, typically beginning with a bacterial oropharyngeal infection, such as pharyngitis or tonsillitis, that results in the spread of the primary infection into the soft tissues. ${ }^{2}$ LS is difficult to diagnose due to its low incidence rates and absence of pathognomonic symptoms. Furthermore, the initial presentation of LS includes general symptoms such as fever, sore throat, bilateral neck swelling, and pulmonary system arthralgia. Given the high prevalence of antibiotic usage, identification of this disease tends to be masked due to antibiotic susceptibility of the most common causative bacteria. Infection can be complicated by thrombophlebitis of the internal jugular vein and septic emboli to the lungs or other organs. ${ }^{3,4}$ Septic pulmonary emboli can occur in up to $97 \%$ of cases of LS, which can be seen with CT scan or chest X-ray, and manifest as chest pain and dyspnea. CT scan of the neck will show occlusion of the internal jugular vein with adjacent swelling of the pharyngeal soft tissue. ${ }^{5}$

The most common causative microorganism is $F$. necrophorum, a component of the oral microbial flora, that is an anaerobic, nonspore-forming, gram negative rod. 1,3 While the pathogenic mechanism of $F$. necrophorum is complex and not well defined, it has been speculated that its leukotoxin and endotoxin, in addition to its ability to aggregate platelets, are the primary virulence factors in overcoming host defense. ${ }^{6}$ Oropharyngeal perforation concomitant with infection can result in local invasion of bacteria through the connective tissue. ${ }^{3}$ If prompt and adequate antibiotic coverage is not obtained, then this infection may spread to distant sites causing further complications such as thrombophlebitis or pleural effusions. ${ }^{6}$

Streptococcus constellatus is a Gram-positive, non-spore forming, non-motile, microaerophilic cocci. It is classified as viridans Streptococcus, and belongs to the $S$. anginosus group, along with $S$. anginosus and $S$. intermedius. All three have been cultured from the oral cavity, intestinal tract, and female urogenital tract. ${ }^{7} S$. constellatus is unique among viridans Streptococci because it is pyogenic and frequently causes abscesses. ${ }^{7} \mathrm{~S}$. constellatus has also been found to have a predilection for the human throat and isolated in pharyngitis patients. ${ }^{7}$

\section{TREATMENT AND MANAGEMENT}

A CT scan of the neck and chest was performed. The CT scan of the neck showed a large fluid and gas collection originating from the left hypopharynx and extending into the left parapharyngeal region. It also showed focal narrowing of the left internal jugular vein with a flow void indicative of thrombus. Dissection in the deep tissues of the neck revealed purulent drainage that was sent for culture. The wound culture grew S. constellatus. The CT scan of the chest also showed extensive subcutaneous emphysema across the midline and into the right neck and upper mediastinum. The patient was immediately given broad spectrum antibiotics to avoid extension of the infection and was taken to the Operating Room (OR) for a direct laryngoscopy.

Once the direct laryngoscopy was performed, it revealed erythematous mucosa with a defect of $3 \mathrm{~cm}$ in the lateral pharyngeal wall. There was no pus or foreign bodies identified in the defect. The laceration was closed with simple interrupted stitches. Then a flexible esophagoscopy was performed, which identified a normal proximal esophagus. A nasogastric tube was then placed. Next, there was an incision made on the left neck crease. The incision was made, and dissection was carried down to the left sternocleidomastoid. The tissue was normal in appearance but had a firm and woody texture. Subsequently, finger dissection was undertaken along the anterior border of the left SCM into the deep tissues of the neck. Copious foul-smelling purulent drainage was identified, and a specimen was sent for culture. Further finger dissection was carried out over the midline and into the right neck, superiorly toward the submentum and inferiorly into the mediastinum. A Pulse-Vac was used to copiously irrigate the wound with antibiotic saline. Of note, no necrotic appearing tissue was encountered. The wound was then packed with iodoform gauze and a dressing was applied over the defect. The patient was then transferred to the ICU on the ventilator. A CT scan of the neck was performed during the hospital course and showed improvement in abscess drainage. 


\section{DISCUSSION}

The most common causative microorganism of LS is F. necrophorum. However, we report a case of LS where the causative bacteria are $S$. constellatus, a member of the $S$. anginosus group, and a common component of the oral flora. The most common manifestations of Anginosus group infections are abscesses in the head, neck, lung, and abdomen. ${ }^{9}$ While the precise virulence factors are not entirely understood, the combination of a mucosal injury in the oropharynx and a bacterial infection could allow local invasion by S. constellatus; which may then have resulted in direct invasion through the connective tissue and spread to other anatomical sites.

\section{CONCLUSIONS}

The pathogenesis of LS is complex and not well defined. What is known is that F. necrophorum is the most common cause of LS. Complications of this disease can be caused by dissemination of septic emboli which travel to major organs and cause damage. Accordingly, prompt diagnosis and treatment of LS is critical to ensure improved patient outcomes. We acknowledge that a case of LS caused by $S$. constellatus, has previously been documented to have a synergistic relationship with Bezold's abscesses in the literature. ${ }^{10}$ However, unique to this case, $S$. constellatus was due to direct laceration of the pharyngeal mucosa. Our case highlights the importance of quick diagnosis of LS and prompt treatment, with rapid identification of the causative bacteria.

\section{Informed Consent}

Informed consent has been obtained from the patient for publication of the case report.

\section{Declaration of Conflicting Interests}

The author(s) declare(s) that there is no conflict of interest.

\section{Funding}

The author(s) received no financial support for the research, authorship, and/or publication of this article.

\section{References}

1. B K, A., Gilotra, T., Tymko, C., Siddique, Z., \& Eranki, A. (2020). A Rare Case of Lemierre's Syndrome Caused by Streptococcus Intermedius, Presenting as an Epidural Abscess. Cureus, 12(4), e7787. https://doi.org/10.7759/cureus.7787

2. Nadkarni MD, Verchick J, O’Neill JC. Lemierre syndrome. J Emerg Med. 2005;28(3):297-9.

3. De Smet K, Claus PE, Alliet G, Simpelaere A, Desmet G. Lemierre's syndrome: a case study with a short review of literature. Acta Clin Belg. 2019:1-5.

4. Srivali N, Ungprasert $P$, Kittanamongkolchai W, Ammannagari N. Lemierre's syndrome: An often missed lifethreatening infection. Indian J Crit Care Med. 2014;18(3):170-2.

5. Charles K, Flinn WR, Neschis DG. Lemierre's syndrome: a potentially fatal complication that may require vascular surgical intervention. J Vasc Surg. 2005;42(5):1023-5.

6. Wani P, Antony N, Wardi M, Rodriguez-Castro CE, Teleb M. The Forgotten One: Lemierre's Syndrome Due to GramNegative Rods Prevotella Bacteremia. Am J Med Case Rep. 2016;17:18-22

7. Faden $\mathrm{H}$, Mohmand M. Infections Associated With Streptococcus Constellatus in Children. Pediatr Infect Dis J. 2017;36(11):1099-100.

8. Stratton CW. Infections Due to the Streptococcus anginosus (Streptococcus milleri) group. In: Sexton DJ, editor. Up To Date. Waltham: Mass; 2017.

9. Bahall M, Giddings S, Bahall K. Lemierre's syndrome: forgotten, but not absent. BMJ Case Rep. 2017;2017.

10. Yaita, K., S. Sugi, M. Hayashi, T. Koga, T. Ebata, Y. Sakai, S. Kaieda, H. Ida, and H. Watanabe. 2018. 'The coexistence of Lemierre's syndrome and Bezold's abscesses due to Streptococcus constellatus: A case report. Medicine (Baltimore), 97: e11228. doi.org/10.1097\%2FMD.0000000000011228 